\title{
Erratum to: The phenomenon of focal segmental glomerulosclerosis post-transplantation-a one-hit wonder?
}

\author{
Moin A. Saleem \\ Published online: 14 August 2012 \\ (C) IPNA 2012

\section{Erratum to: Pediatr Nephrol \\ DOI 10.1007/s00467-012-2218-5}

Unfortunately the copy editor introduced errors into the abstract. The publisher apologizes for this mishap and is pleased to reproduce the correct abstract in full.

\begin{abstract}
Steroid resistant nephrotic syndrome (SRNS), otherwise termed focal segmental glomerulosclerosis (FSGS) is one of the most difficult conditions to manage for the nephrologist, particularly when the disease recurs post-transplantation. It is also fascinating from the biological perspective, as the non-genetic form appears highly likely to be caused by a disorder of circulating plasma, leading to the search for the elusive 'plasma factor' over several decades of research. Many hypotheses have been proposed and tested, and to date none have yet passed the test of clinical utility. The search appears to be narrowing, aided by landmark discoveries in the molecular properties of the glomer-
\end{abstract}

ular filtration barrier, and improved experimental tools. Therapeutically we are also more able to target specific molecules, for example by monoclonal antibody treatments. In this context, the report of the effects of TNF- $\alpha$ on podocytes is instructive. This tells us that this cytokine could have directly deleterious effects on podocytes in vivo, and that this effect can be targeted clinically, potentially halting or reversing the disease process. As with all thought provoking research, this raises several interesting questions. Is TNF- $\alpha$ the elusive 'factor' or is it one of several? Is it directly affecting the glomerular filtration barrier, or modulating the immune response? And could this technique be used as a cellbased assay for disease activity? This report adds to the growing list of candidates that need to be tested in a wider population of well phenotyped patients with SRNS.

Keywords Steroid resistant nephrotic syndrome - Focal segmental glomerulosclerosis - Plasma factor . TNF- $\alpha$. Podocyte $\cdot$ Proteinuria $\cdot$ Cytokine $\cdot$ Cytoskeleton

The online version of the original article can be found at http://dx.doi.org/ 10.1007/s00467-012-2218-5.

M. A. Saleem $(\square)$

Children's and Academic Renal Unit, Southmead Hospital-

University of Bristol,

Bristol BS10 5NB, UK

e-mail:m.saleem@bristol.ac.uk 